\title{
Incidencia, factores de riesgo y tratamiento de las complicaciones biliares del trasplante hepático*
}

\author{
Drs. MAURICIO GABRIELLI N. ${ }^{1}$, ENRIQUE NORERO M. ${ }^{1}$, EDUARDO FIGUEROA R. ${ }^{1}$, \\ PABLO CORTÉS G. ${ }^{2}$, FERNANDO PIMENTEL M. ${ }^{1}$, ÁLVARO HUETE G. ${ }^{3}$, \\ LUIS MENESES Q. ${ }^{3}$, SOLEDAD LOYOLA Z. ${ }^{3}$, MARCO ARRESE J. ${ }^{2}$, \\ ALEJANDRO SOZA R. ${ }^{2}$, ROSA MARÍA PÉREZ A. ${ }^{2}$, E.U. PILAR DOMÍNGUEZ B. ${ }^{1}$, \\ Drs. JUAN FRANCISCO GUERRA C. ${ }^{1}$, NICOLÁS JARUFE C. ${ }^{1}$, JORGE MARTÍNEZ C. ${ }^{1}$ \\ Departamento de Cirugía Digestiva. \\ Departamento de Gastroenterología. \\ Departamento de Radiología. \\ Hospital Clínico, Facultad de Medicina. Pontificia Universidad Católica de Chile. \\ Santiago, Chile.
}

\begin{abstract}
Biliary tract complications after liver transplantation

Introduction: Biliary tract complications (BC) are cause of mortality after liver transplantation (LT). There are different treatment alternatives for this complication. Aim: to determinate incidence, risk factors and treatment of biliary complications after LT. Materials and Methods: A retrospective descriptive cohort of patients undergoing LT between March 1994 and March 2009. Risk factors and incidence for BC were explored. Also the BC impact on overall survival on LT patients was assessed. We used SPSS 15.0 for statistical analysis and considered a significant $\mathrm{p}$ value less than 0.05 . Results: 107 LT were performed in 102 patients. In $30(28 \%)$ there was some biliary complication. Ten (33.3\%) were early complications $(<3$ months) and 20 $(66.7 \%)$ were late ( $\geq 3$ month). Anastomotic stricture was the more frequent $\mathrm{BC}$. The gender male recipient, the cold ischemic time and biliary reconstruction technique without tutor were associated with an increased risk for BC. Endoscopic treatment of biliary stricture was successful in $91 \%$ of cases at one year follow up. Three $(10 \%)$ patients died due to $\mathrm{BC}$ or their long-term treatment. Conclusions: Biliary Complications after Liver Transplantation are frequent and are cause of mortality. Decrease cold ischemic time and improve techniques for biliary reconstruction could reduce the high incidence of this complication.
\end{abstract}

Key words: Liver transplantation, biliary complications, risk factors.

\section{Resumen}

Introducción: Las complicaciones biliares (CB) en el trasplante hepático (TH) son causa de mortalidad post-trasplante. Existen diferentes alternativas terapéuticas para su tratamiento. Objetivo: Determinar la incidencia, factores de riesgo y evaluar los resultados del tratamiento de las CB posterior al TH. Materiales

\footnotetext{
*Recibido el 4 de Febrero de 2010 y aceptado para publicación el 17 de Marzo de 2010.

Correspondencia: Dr. Jorge Martínez C.

Marcoleta 352, Santiago, Chile. Fax: 56-2-638-2793.

E-mail: jmartinez@med.puc.cl
} 
y Método: Estudio retrospectivo, descriptivo de una cohorte de pacientes sometidos a un TH entre marzo-1994 y marzo-2009. Se analizó los factores de riesgo para CB, su incidencia en el tiempo, su tratamiento y se determinó su impacto en la sobrevida global de los pacientes. Se utilizó el programa SPSS 15.0 para cálculos estadísticos y se consideró significativo un $\mathrm{p}<0,05$. Resultados: Se realizaron $107 \mathrm{TH}$ en 102 pacientes. En 30 (28\%), se consignó una o más CB. En 10 (33,3\%), la CB fue precoz y en 20 (66,7\%) tardía. La estenosis de la anastomosis fue la CB más frecuente. El sexo masculino del receptor, el tiempo de isquemia fría y la técnica de reconstrucción biliar sin tutor, se asociaron significativamente a un mayor riesgo de CB. El tratamiento endoscópico de la estenosis biliar fue exitoso en el $91 \%$ de los casos a un año de seguimiento. Tres (10\%) pacientes fallecieron por una CB o su tratamiento a largo plazo. Conclusiones: Las CB del TH son frecuentes y son causa de mortalidad. Disminuir el tiempo de isquemia fría y mejorar las técnicas de reconstrucción biliar podrían disminuir su incidencia.

Palabras clave: Trasplante hepático, complicaciones biliares, factores de riesgo.

\section{Introducción}

Las complicaciones derivadas de la reconstrucción de la vía biliar son consideradas, aún hoy, el talón de Aquiles del trasplante hepático. Starzl y $\operatorname{cols}^{1}$, en las primeras comunicaciones informaron una mortalidad de hasta un $30 \%$, secundaria a éstas. Hoy en día, debido a los avances en su tratamiento, los casos de mortalidad no superan el $10-15 \%^{2}$; sin embargo, la morbilidad es aún relevante con una incidencia que varía entre el 10 y el $30 \% \%^{3,4}$. Estas altas tasas de $\mathrm{CB}$ posterior al $\mathrm{TH}$, alertan acerca de la inherente sensibilidad del epitelio biliar al daño por isquemia, en comparación al hepatocito o al endotelio vascular ${ }^{5}$. Las estenosis y filtraciones son las CB más frecuentes. Éstas se pueden presentar en cualquier etapa del trasplante y con frecuencia su cuadro clínico se puede confundir con otras complicaciones infecciosas o inmunológicas del trasplante ${ }^{6}$.

Pese a los avances en la técnica quirúrgica y cuidados peri-operatorios del paciente trasplantado, las $\mathrm{CB}$ constituyen un desafío terapéutico para los equipos tratantes. Trabajos recientes, señalan al manejo endoscópico como una alternativa atrayente en el tratamiento de estos pacientes al ser menos invasivo, de utilidad diagnóstica y terapéutica y al tener una baja morbilidad en centros especializados ${ }^{7,8}$.

El objetivo de este estudio, fue determinar la incidencia de las complicaciones biliares posterior al trasplante hepático en el Hospital Clínico de la Pontificia Universidad Católica (HC de la PUC), explorar sus eventuales factores de riesgo, evaluar los resultados de su tratamiento y determinar su impacto en la sobrevida de injertos y pacientes.

\section{Material y Método}

La información fue obtenida de los registros clínicos y de una base de datos prospectiva de todos los pacientes que ingresaron al Programa de Trasplante
Hepático del HC de la PUC entre marzo de 1994 y marzo de 2009. El seguimiento promedio de la serie fue de 3 años. Todos los trasplantes fueron realizados con la técnica de Piggy-back modificada, descrita previamente 9 . Las técnicas de reconstrucción biliar realizadas se resumen en la Tabla 1.

La solución de preservación utilizada en la cirugía de obtención del injerto fue la de la Universidad de Wisconsin (UW) (Viaspán ${ }^{\mathrm{R}}$ ) desde 1994 a 2003 y desde entonces, en forma aleatoria, la solución Histidina-Triptofano-Ketoglutarato (HTK) $\left(\right.$ Custodiol $\left.^{\mathrm{R}}\right) \mathrm{o}$ UW. En todos se utilizó un esquema inmunosupresor primario bi o triasociado sobre la base de un anticalcineurínico (ciclosporina o tacrolimus) y esteroides, acompañados de un antiproliferativo (azatioprina o micofenolato mofetil) en los esquemas triasociados. Éstos fueron readecuados de acuerdo a seguimiento clínico y a los diferentes protocolos de inmunosupresión utilizados en el tiempo.

Se determinaron las características demográficas de los donantes y de los receptores. Los siguientes factores de riesgo conocidos para el desarrollo de una complicación biliar en TH fueron explorados: edad del donante, tiempo de isquemia fría, tipo de reconstrucción biliar, sexo y edad del receptor. Se analizaron por separado aquellos pacientes que presentaron alguna complicación vascular de manifestación biliar. Las CB se clasificaron según el momento

Tabla 1. Técnicas de reconstrucción biliar usadas en los 107 trasplantes hepáticos

\begin{tabular}{lcc}
\hline Técnica & n de casos & $(\mathbf{\%})$ \\
\hline TT tutor transcístico & 59 & $(55)$ \\
TT sin tutor & 19 & $(19)$ \\
Y de Roux & 11 & $(10)$ \\
TT tutor interno doble pig tail & 9 & $(8)$ \\
TT con sonda T & 9 & $(8)$ \\
\hline
\end{tabular}

TT: término-terminal. 
de su diagnóstico en precoces (perioperatorias y $\leq$ de 3 meses) y tardías ( $>3$ meses). Para el cálculo de la tasa de éxito del tratamiento de la CB se utilizó un año de seguimiento. El impacto de la CB en la sobrevida global de los pacientes se calculó con el método de Kaplan-Meier. El cierre del estudio fue marzo de 2009. Para cálculos estadísticos se utilizó el programa informático SPSS 15.0. Se consideró significativo un valor de $\mathrm{p}<0,05$.

\section{Resultados}

En el período del estudio se realizaron 107 trasplantes hepáticos en 102 pacientes. La edad promedio de los enfermos fue de $48 \pm 15$ años; 64 (57\%) fueron hombres y 102 trasplantes (95\%) fueron realizados en adultos. En 28 (26\%) casos la indicación del trasplante hepático fue de urgencia. La Tabla 2 muestra la etiología del daño hepático que fue la indicación del trasplante, por frecuencia. Se observa que las indicaciones más frecuentes fueron el daño hepático crónico (cirrosis) por alcohol y por virus $\mathrm{C}$.

En $30(28 \%)$ pacientes se consignó alguna complicación biliar; éstas se detallan en la Tabla 3. En 10 casos $(33,3 \%)$ la $\mathrm{CB}$ fue de manifestación precoz y en $20(66,7 \%)$ tardía, siendo la estenosis de la anastomosis la CB más frecuente. Además se consignaron dos pacientes con una complicación vascular de manifestación biliar: uno de ellos con una trombosis de la arteria hepática y el otro con una estenosis del tronco celíaco (Figura 1A).

Ambos pacientes se presentaron con una estenosis anastomótica biliar precoz (Figura 1B).

\section{Factores de riesgo de complicación biliar}

Un análisis univariado de varios factores explorados mostró que el sexo masculino del receptor $(\mathrm{p}=0,003)$, el tiempo de isquemia fría $(\mathrm{p}=0,018)$ y la técnica de reconstrucción biliar término-terminal sin tutor $(p=0,04)$ se asociaron significativamente a un mayor riesgo de complicación biliar (Tabla 4).

\section{Tratamiento de las complicaciones biliares del trasplante hepático}

En 8 de 19 pacientes que presentaron una estenosis anastomótica de la vía biliar, se realizó una derivación biliodigestiva con una asa yeyunal desfuncionalizada en "Y de Roux" como tratamiento de primera línea. La cirugía biliar reconstructiva fue exitosa en el $100 \%$ de los pacientes al año de seguimiento. Uno de ellos, desarrolló una estenosis segmentaria del conducto biliar izquierdo a los 2 años de la derivación biliodigestiva (Figura 2), con cuadros de colangitis aguda a repetición. Luego de dos intentos frustros de tratamiento percutáneo y endoscópico respectivamente, fue sometido a una resección hepática de los segmentos 2 y 3 de su injerto y evolucionó en forma favorable ${ }^{10}$.

En $10(91 \%)$ de 11 pacientes el tratamiento endoscópico, con instalación progresiva de múltiples prótesis cada 3 meses fue exitoso. El paciente restante, se perdió de controles y a los 6 meses consultó por colestasia y recurrencia de una obesidad mórbida (IMC: 34,5$)$, siendo sometido a una reconstrucción biliar quirúrgica con un asa en $\mathrm{Y}$ de Roux, y a una gastrectomía vertical en manga realizadas en el mismo acto quirúrgico ${ }^{11}$. No hubo tratamiento percutáneo ni retrasplante en el grupo de $\mathrm{CB}$ estenóticas.

Tabla 2. Indicaciones de 107 trasplantes hepáticos en 102 pacientes

\begin{tabular}{lcc}
\hline Etiología & n & $(\mathbf{\% )}$ \\
\hline Alcohol & 19 & $(17,8)$ \\
Virus hepatitis C & 18 & $(16,8)$ \\
Criptogénica & 16 & $(14,9)$ \\
NASH & 15 & $(14)$ \\
HAI & 11 & $(10,4)$ \\
Cirrosis biliar primaria & 8 & $(7,6)$ \\
Hemocromatosis & 7 & $(6,5)$ \\
Retrasplante & 5 & $(4,7)$ \\
Metástasis neuroendocrina & 4 & $(3,7)$ \\
Virus hepatitis A & 2 & $(1,8)$ \\
Drogas & 2 & $(1,8)$ \\
\hline
\end{tabular}

NASH: esteatohepatitis no alcohólica. HAI: hepatitis autoinmune.

Tabla 3. Complicaciones biliares precoces y tardías posterior a trasplante hepático

\begin{tabular}{lcl}
\hline $\begin{array}{l}\text { Momento de } \\
\text { complicación (n) }\end{array}$ & $\begin{array}{c}\text { n de } \\
\text { casos }\end{array}$ & Tipo \\
\hline $\begin{array}{l}\text { Complicación } \\
\text { biliar precoz (10) }\end{array}$ & & \\
Intrahospitalaria & 3 & Estenosis anastomosis \\
& 3 & Bilioma \\
$\leq 3$ meses & 4 & Estenosis anastomosis \\
Complicación & & \\
biliar tardía (20) & & \\
$>3$ meses y & 6 & Estenosis anastomosis \\
$\leq 12$ meses & & \\
& 3 & $\begin{array}{l}\text { Estenosis no anastomótica } \\
\text { Filtración por retiro sonda }\end{array}$ \\
$>12$ meses & 3 & $\begin{array}{l}\text { Estenosis anastomosis } \\
\end{array}$ \\
& 3 & Bilioma \\
& 2 & Coledocolitiasis \\
\hline
\end{tabular}




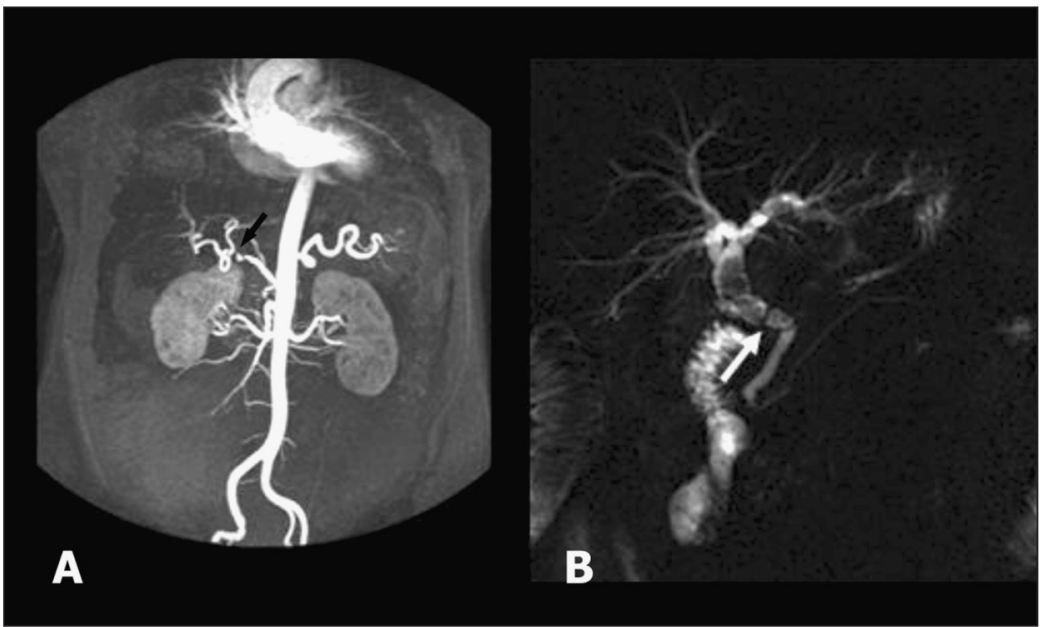

Figura 1. A. Angiografría por resonancia nuclear magnética en donde se observa la estenosis a nivel de la anastomosis en la arteria hepática común (flecha negra). B. Colangiografía por resonancia magnética en donde se observa la estenosis anastomótica de la vía biliar (flecha blanca) del mismo paciente.

Tabla 4. Análisis univariado de los factores de riesgo para complicación biliar

\begin{tabular}{lccc}
\hline Factor de riesgo & Sin complicación biliar & Con complicación biliar & Valor-p \\
\hline Sexo masculino & $33 \%$ & $73 \%$ & 0,003 \\
Edad receptor & $44 \pm 14$ años & $45 \pm 14$ años & NS \\
Edad donante & $36 \pm 15$ & $38 \pm 15$ & NS \\
Tiempo isquemia fría & $445 \pm 170$ min & $610 \pm 200$ min & 0,018 \\
Anastomosis TTST & $45 \%$ & $62 \%$ & 0,04 \\
\hline
\end{tabular}

TTST: término terminal sin tutor.

De los 9 pacientes que presentaron una filtración biliar asociada o no a una estenosis biliar, se realizó tratamiento percutáneo en 4, tratamiento endoscópico en 3 y 2 se reoperaron realizándose una derivación bilio-digestiva con una $\mathrm{Y}$ de Roux.

Uno de los 3 pacientes con filtración biliar tras el retiro del drenaje de Kehr a los 3 meses del trasplante, presentó un biliperitoneo pese al tratamiento endoscópico con una papilotomía sin instalación de prótesis, debiendo ser reoperado para aseo y drenaje.

Los 2 pacientes en que la complicación biliar fue considerada una manifestación de una complicación vascular, fueron operados. En uno, se practicó una revascularización quirúrgica con un puente protésico de PTFE entre la aorta del receptor y la arteria hepática común del injerto, que fue seguida de una reconstrucción biliar con una Y de Roux. Sin embargo, pese a una evolución inicial favorable, a los 8 meses después requirió de un retrasplante por evolución hacia estenosis múltiples y difusas intra-hepáticas, con su puente vascular permeable; el otro enfermo con estenosis de la anastomosis arterial en el tronco celíaco del donante, fue tratado

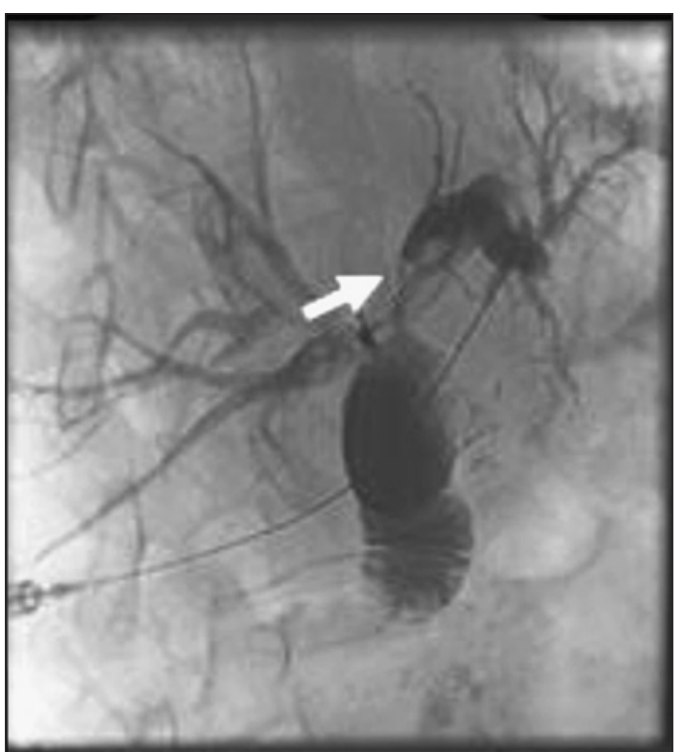

Figura 2. Colangiografía directa percutánea transparietohepática en donde se observa una estenosis intrahepática del conducto biliar izquierdo, proximal a la anastomosis bilio-entérica. 


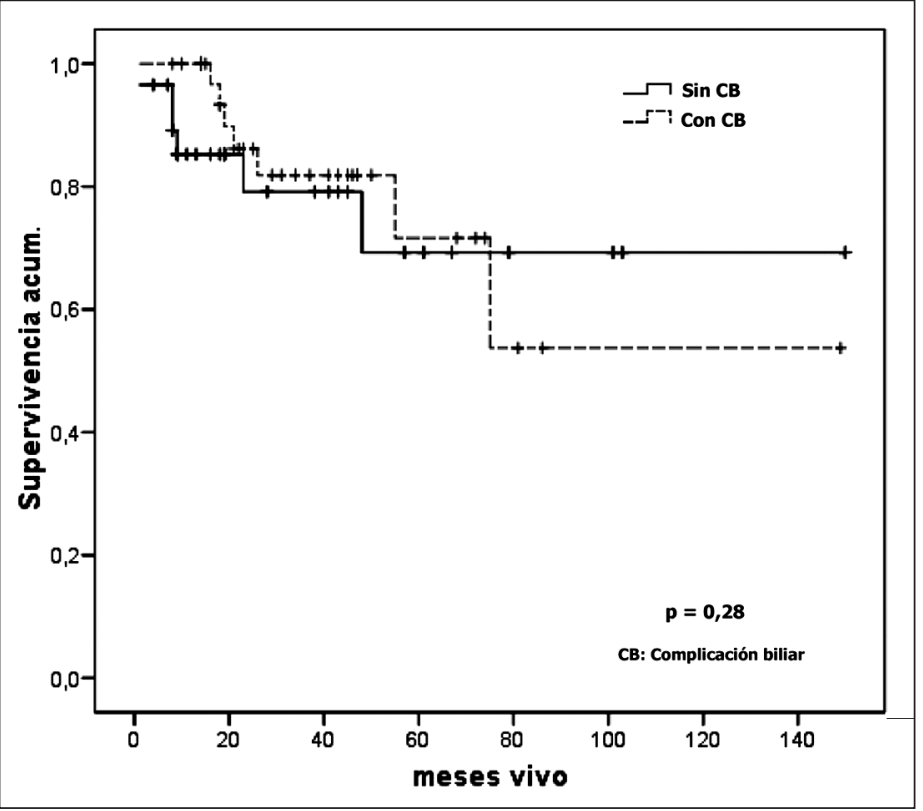

Figura 3. Sobrevida global de 102 pacientes con trasplante de hígado según la presencia (con CB) o ausencia (sin CB) de complicaciones biliares. Nótese que la sobrevida a 5 años de los pacientes con y sin complicaciones biliares es superior al $75 \%$.

con un tutor instalado por vía endovascular, lo que fue seguido de una reconstrucción biliar con un asa yeyunal desfuncionalizada. Actualmente el paciente está en controles periódicos con pruebas de función hepática normales.

Tres pacientes $(10,7 \%)$ fallecieron en relación a una complicación biliar tardía o a su tratamiento después del año del trasplante; todos ellos tratados en sus hospitales de origen. La sobrevida global a 5 años de los enfermos no mostró diferencias significativas según la presencia o no de complicación biliar (Figura 3).

\section{Discusión}

Esta serie es la primera publicación nacional enfocada a las complicaciones biliares posterior al trasplante hepático, pese a que en la literatura internacional éstas constituyen una entidad ampliamente comunicada $^{3,4,12}$. Existe un claro consenso en que las complicaciones biliares del trasplante hepático continúan representando su talón de Aquiles, provocando incluso mortalidad en el paciente trasplantado $^{13}$. Otra serie nacional coincide con cifras de CB de un $29 \%{ }^{14}$, aún con un menor tiempo de isquemia fría que la presente. Estas cifras de CB están entre las altas comunicadas, si bien en las diversas publicaciones el seguimiento es menor que el nuestro ${ }^{4,13}$. Afortunadamente, ellas no determinaron un impacto mayor en la sobrevida global de los pacientes de toda la serie, pese a que se asoció a mortalidad en 3 pacientes. Nos parece importante señalar que esos pacientes fallecidos fueron tratados en sus hospitales de origen de acuerdo a las normas actuales de la autoridad sanitaria y sería deseable que este tipo de complicaciones, aún tardías, sean tratadas más bien en el centro trasplantador. Se trata de pacientes con complicaciones complejas que requieren de un equipo multidisciplinario entrenado en la patología asociada al trasplante para su mejor tratamiento; que no siempre está disponible en todos los hospitales.

El riesgo de una complicación biliar se relaciona entre otros factores con la técnica quirúrgica de reconstrucción ${ }^{15}$, con el uso o no de drenaje biliar ${ }^{16}$, al tipo de hígado trasplantado ${ }^{17}$, a los tiempos de isquemia $^{18}$ y a las características del donante y el receptor ${ }^{19}$. Los injertos parciales, sea por reducción para receptores pequeños o por la utilización de hígados divididos (SPLIT) para 2 receptores simultáneos o aquellos obtenidos de donantes vivos se asocian a una mayor incidencia de $\mathrm{CB}$ en relación a injertos completos de donantes cadavéricos ${ }^{20}$. Además, varios autores han demostrado que el tiempo de isquemia fría prolongado es un factor de riesgo independiente para el desarrollo de estenosis de la vía biliar ${ }^{21,22}$.

En esta serie, al igual que en otras ${ }^{24,25}$, la estenosis de la vía biliar fue la complicación biliar más frecuente. La estenosis a nivel de la anastomosis fue la ubicación más habitual (84,2\%). Estas complicaciones de acuerdo a otros autores serían principalmente de manifestación precoz ${ }^{4,26}$. No obstante, en este estudio, más del 50\% de ellas fueron de manifestación 
tardía. Esto sugiere que la cicatrización fibrótica de la anastomosis biliar, enlentecida por el uso de inmunosupresores, tendría un rol significativo en el desarrollo de la estenosis de la anastomosis biliar tardía como ya ha sido descrito por otros ${ }^{27,28}$. Estos casos, no serían atribuibles a problemas o a isquemia prolongada del árbol biliar. Por el contrario, en las filtraciones y estenosis precoces, ambos factores sí tienen un rol relevante en su incidencia ${ }^{29}$.

Actualmente no existe consenso sobre la mejor alternativa terapéutica para las $\mathrm{CB}$ del $\mathrm{TH}$; sin embargo, el tratamiento endoscópico es atractivo al ser de utilidad diagnóstica y terapéutica con un bajo riesgo, en especial en el tratamiento de las anastomosis conducto a conducto término-terminal ${ }^{30}$. El uso de múltiples prótesis seriadas ha mejorado los resultados del manejo endoscópico de la estenosis anastomótica biliar ${ }^{31,32}$. Morelli y cols, informaron una tasa de éxito de un $88 \%$ al año de seguimiento con esta modalidad de tratamiento, cifra que concuerda con los resultados de nuestra serie ${ }^{32}$. El mismo autor, en otro estudio, demostró mejoría en los resultados con un protocolo acelerado de dilataciones endoscópicas cada dos semanas ${ }^{33}$. En esta serie, los ocho pacientes con estenosis de la vía biliar que se trataron primariamente con una derivación bilio-digestiva, fueron trasplantados al inicio del programa, cuando el manejo endoscópico seriado aún estaba en implementación o presentaron concomitantemente otra complicación biliar asociada: bilioma y/o filtración.

La técnica de reconstrucción biliar sin tutor es un factor de riesgo para estenosis de la vía biliar, sin embargo, se debe considerar que la decisión de no usar tutor durante la reconstrucción de la vía biliar va en estrecha relación con el diámetro de ésta. El tiempo de isquemia fría y el sexo masculino resultaron ser factores de riesgo para complicación biliar, situación que coincide con lo informado en la literatura ${ }^{34}$. En relación al tipo de solución de preservación, ésta no se incluyó en el análisis univariado debido al menor número de pacientes en que la solución de preservación utilizada fue el HTK, lo que le quitaba fuerza estadística a esta información. La literatura, no es concluyente en si este factor incide mayormente en la frecuencia de $\mathrm{CB}$, si bien tiende a señalar que la mayor viscosidad de la solución UW puede comprometer el lavado de la red arteriolar peri-biliar contribuyendo a un incremento en las estenosis biliares ${ }^{35}$.

En el análisis de sobrevida alejada, las curvas no son estadísticamente diferentes $(\mathrm{p}=0,280)$, sin embargo, luego de los 6 años parece observarse una menor sobrevida en los pacientes que tuvieron alguna $\mathrm{CB}$.

En conclusión, las complicaciones biliares conti- núan siendo un problema frecuente en el trasplante hepático y su apropiado tratamiento requiere del concurso de diversos especialistas y una discusión multidisciplinaria caso a caso, idealmente en su propio centro trasplantador.

\section{Referencias}

1. Starzl TE, Putnam CW, Hansbrough JF, Porter KA, Reid HA. Biliary complications after liver transplantation with special reference to the biliary cast syndrome and techniques of secondary duct repair. Surgery 1977; 81: 212-221.

2. Welling TH, Heidt DG, Englesbe MJ, Magee JC, Sung RS, Campbell DA, et al. Biliary complications following liver transplantation in the model for end-stage liver disease era: effect of donor, recipient, and technical factors. Liver Transpl 2008; 14: 73-80.

3. Greif F, Bronsther OL, Van Thiel DH, Casavilla A, Iwatsuki S, Tzakis A. The incidence, timing, and management of biliary tract complications after orthotopic liver transplantation. Ann Surg 1994; 219: 40-45.

4. O'Connor TP, Lewis WD, Jenkins RL. Biliary tract complications after liver transplantation. Arch Surg 1995; 130: 312-317.

5. Noack K, Bronk SF, Kato A, Gores GJ. The greater vulnerability of the bile duct cells to reoxygenation injury than to anoxia. Implications for the pathogenesis of biliary strictures after liver transplantation. Transplantation 1993; 56: 495-500.

6. Busuttil R, Farmer D, Yersiz H, Hiatt JR, McDiarmid $\mathrm{SV}$, Goldstein LI, et al. Analysis of long-term outcomes of 3200 liver transplantations over two decades: a single-center experience. Ann Surg 2005; 241: 905-916.

7. Holt AP, Thorburn D, Mirza D, Gunson B, Wong T, Haydon G. A prospective study of standardized nonsurgical therapy in the management of biliary anastomotic strictures complicating liver transplantation. Transplantation 2007; 84: 857-863.

8. Londono MC, Balderramo D, Cárdenas A. Management of biliary complications after orthotopic liver transplantation: the role of endoscopy. World Gastroenterol 2008; 14: 493-497.

9. Martínez J. Implantación con técnica de Piggy-Back y anastomosis cavo-cava latero-lateral en trasplante hepático ortotópico. Experiencia inicial en un Centro universitario. Rev Chil Cir 2004; 56: 317-331.

10. Guerra JF, Jarufe N, Martínez J. Hepatic resection after liver transplantation as a graft-saving procedure. Transplantation Proceedings 2009; 41: 1994-1996.

11. Butte JM, Devaud N, Jarufe N, Boza C, Pérez G, Torres $\mathrm{J}$, et al. Sleeve gastrectomy as treatment for severe obesity after orthotopic liver transplantation. Obes Surg 2007; 17: 1517-1519.

12. Stratta RJ, Wood RP, Langnas AN, Hollins RR, Bruder 
KJ, Donovan JP, et al. Diagnosis and treatment of biliary tract complications after orthotopic liver transplantation. Surgery 1989; 106: 675-683.

13. Safdar K, Atiq M, Stewart C, Freeman ML. Biliary tract complications after liver transplantation. Gastroenterol Hepatol 2009; 3: 183-195.

14. Hepp J, Ríos H, Suárez L, Zaror M, Quiroga M, Rodríguez G, y cols. Trasplante hepático en adultos: casuística de Clínica Alemana de Santiago. Rev Med Chile 2002; 130: 779-786.

15. Sanni A, Asher J, Wilson C, Wyrley-Birch H, Vijayanand $\mathrm{D}$, Jaques $\mathrm{B}$, et al. Predisposing factors for biliary complications following liver transplantation. Transplant Proc 2006; 38: 2677-2678.

16. Wojcicki M, Lubikowski J, Klek R, Post M, Jarosz K, Białek A, et al. Reduction of biliary complication rate using continuous suture and no biliary drainage for duct-to-duct anastomosis in whole-organ liver transplantation. Transplant Proc 2009; 41: 3126-3130.

17. Sharma S, Gurakar A, Jabbour N. Biliary strictures following liver transplantation: past, present and preventive strategies. Liver Transpl 2008; 14: 759-769.

18. D'Alessandro AM. Normothermic liver preservation: is it time to come in from the cold?. Ann Surg 2009; 250: 7-9.

19. Macedo FI, Miranda LE, Pádua TC, Fernandes JL, Neto OL, Lacerda CM. Effects of donor age on patient survival in liver transplantation: short-and long-term analysis. Hepatogastroenterology 2009; 56: 1133-1136.

20. Wilms C, Walter J, Kaptein M, Mueller L, Lenk C, Sterneck M, et al. Long-term outcome of split liver transplantation using right extended grafts in adulthood: A matched pair analysis. Ann Surg 2006; 244: 865-872.

21. Berm J, Hyuck C, Choi GS, Chun J, Jung G, Kim S-J, et al. Prolonged cold ischemic time is a risk factor for biliary strictures in duct-to-duct biliary reconstruction in living donor liver transplantation. Transplantation 2008; 86: $1536-1342$.

22. Sánchez-Urdazpal L, Gores G, Mard E, Maus T, Wahlstrom E, Moore S. Ischemic-type biliary complications after orthotopic liver transplantation. Hepatology 1992; 16: 49-53.

23. Wojcicki M, Milkiewicz P, Silva M. Biliary Tract complications after liver transplantation: A review. Dig Surg 2008; 25: 245-257.
24. Colonna JO, Shaked A, Gomes AS, Colquhoun SD, Jurim O, McDiarmid SV, et al. Biliary strictures complicating liver transplantation. Incidence, pathogenesis, management and outcome. Ann Surg 1992; 216: 344350.

25. Yuan D, Wei YG, Lin HM, Li FQ, Yang M, Liu XL. Risk factors of biliary complications following liver transplantation: retrospective analysis of a single centre. Postgrad Med J 2009; 85: 119-123.

26. Sharma S, Gurakar A, Jabbour N. Biliary strictures following liver transplantation: past, present and preventive strategies. Liver Transpl 2008; 14: 759-769.

27. Ostroff JW. Post-transplant biliary problems. Gastrointest Endosc Clin N Am 2001; 11: 163-183.

28. Pascher A, Neuhaus P. Biliary complications after deceased-donor orthotopic liver transplantation. J Hepatobiliary Pancreat Surg 2006; 13: 487-496.

29. Koneru B, Sterling MJ, Bahramipour PF. Bile duct strictures after liver transplantation: a changing landscape of the Achilles' heel. Liver Transpl 2006; 12: 702-704.

30. Williams E, Draganov P. Endoscopic management of biliary strictures after liver transplantation. World $\mathrm{J}$ Gastroenterol 2009; 15: 3725-3733.

31. Costamagna G, Pandolfi M, Multignani M, Spada C, Perri V. Long-term results of endoscopic management of postoperative bile duct strictures with increasing numbers of stentes. Gastrointest Endosc 2001; 54: 162 168.

32. Morelli J, Mulcahy HE, Willner IR, Cunningham JT, Draganov P. Long-term outcomes for patients with postliver transplant anastomotic biliary strictures treated by endoscopic stent placement. Gastrointest Endosc 2003; 58: 374-379.

33. Morelli G, Fazel A, Judah J, Pan JJ, Forsmark C, Draganov P. Rapid-sequence endoscopic management of posttransplant anastomotic biliary strictures. Gastrointest Endosc 2008; 67: 879-885.

34. Tekin K, Imber C, Atli M, Gunson B, Bramhall S, Mayer D. A simple scoring system to evaluate the effects of cold ischemia on marginal liver donors. Transplantation 2004; 77: 411-416.

35. Feng L, Zhao N, Yao X, Sun X, Du L, Diao X, et al. Histidine-tryptophan-ketoglutarate solution $v s$ University of Wisconsin solution for liver transplantation: a systematic review. Liver Transpl 2007; 13: 1125-1136. 